Tetrahedron

journal homepage: www.elsevier.com

\title{
Bimetallic Co-Pd Alloy Nanoparticles as Magnetically Recoverable Catalysts for the Aerobic Oxidation of Alcohols in Water
}

\author{
Yoshikazu Ito, ${ }^{\mathrm{a}, \mathrm{b}}$ Hidetoshi Ohta, ${ }^{\mathrm{a}}$ Yoichi M. A. Yamada, ${ }^{\mathrm{a}}$ Toshiaki Enoki, ${ }^{\mathrm{b}}$ and Yasuhiro Uozumi ${ }^{\mathrm{a}, \mathrm{c}^{*}}$ \\ ${ }^{a}$ RIKEN Center for Sustainable Resource Science, Wako, Saitama 351-0198, Japan \\ ${ }^{b}$ Department of Chemistry, The Graduate School of Science and Engineering, Tokyo Institute of Technology, Ookayama, Tokyo 152-8551, Japan \\ 'Institute for Molecular Science, Myodaiji, Okazaki 444-8787, Japan
}

\section{ARTICLE INFO}

\section{ABSTRACT}

Article history:

Received

Received in revised form

Accepted

Available online

Keywords:

bimetallic catalyst

cobalt-palladium

magnetic nanoparticle

aerobic oxidation

water

\section{Introduction}

The development of heterogeneous catalysts for the aerobic oxidation of alcohols in water is an important topic for pure and industrial chemistry. ${ }^{1}$ For example, we ${ }^{2}$, Kaneda et al. ${ }^{3}$, Sheldon et al. ${ }^{4}$, Corma et al. ${ }^{5}$, and Sakurai et al..$^{6}$ developed non-magnetic heterogeneous nanoparticle catalysts for the aerobic oxidation of various alcohols in water as recoverable catalysts. Recent attention has focused on magnetically recoverable heterogeneous catalysts that can be readily collected by magnetic attraction: metal species supported on magnetic solids have been prepared for the aerobic oxidation of alcohols as magnetically recoverable catalysts. ${ }^{7-12} \mathrm{Fe}_{2} \mathrm{O}_{3}$-immobilized Pd nanoparticles, ${ }^{7}$ mesoporous silica-immobilized $\mathrm{Fe}_{2} \mathrm{O}_{3},{ }^{8} \mathrm{Fe}_{3} \mathrm{O}_{4}$-immobilized $\mathrm{Ru}$ nanoparticles, ${ }^{9}$ $\mathrm{Fe}_{3} \mathrm{O}_{4}$-immobilized $\mathrm{Au}$ nanoparticles, ${ }^{10} \quad \mathrm{Fe}_{3} \mathrm{O}_{4}$-immobilized $\mathrm{Ru}(\mathrm{OH})_{\mathrm{x}}{ }^{11}$ and $\mathrm{NiFe}_{2} \mathrm{O}_{4}$-immobilized $\mathrm{Ru}$ nanoparticles ${ }^{7,}{ }^{12}$ are typical examples with which the oxidation was carried out in $\mathrm{H}_{2} \mathrm{O}_{2}$ in $\mathrm{H}_{2} \mathrm{O}^{7,8}$, polyglycol ${ }^{8}$, toluene ${ }^{9-11}$ and isopropanol ${ }^{12}$ at $70-$ $130{ }^{\circ} \mathrm{C}$. In these materials, non-magnetic catalytic species are supported on non-catalytic magnetic nanoparticles.
Although these pioneering works are valuable, it is important to develop bifunctional bimetallic alloy nanoparticles in which multiple functions of both catalytic and magnetic properties are simultaneously provided by utilizing surface and ligand modification. ${ }^{13}$ Therefore, we decided to develop fine bimetallic alloy magnetic nanoparticles having a strong magnetic moment and high catalytic activity for the aerobic oxidation of alcohols in water. Since Pd nanoparticle catalysts are paramagnetic, ${ }^{14}$ ferromagnetic sources should be combined for magnetizing Pd nanoparticles. Recently, we reported ferromagnetic Pd alloy nanoparticles of $\mathrm{Co}-\mathrm{Pd}^{15}, \mathrm{Fe}-\mathrm{Pd}^{16}$ and $\mathrm{Ni}-\mathrm{Pd}^{17}$ with giant magnetic moment. According to our previous reports, Co-Pd alloy nanoparticles have the strongest giant magnetic moment enhanced by $\mathrm{Co}$ atoms that attract a neodymium $(\mathrm{Nd})$ magnet. We envisioned that bifunctional and bimetallic Co-Pd alloy nanoparticles having a high surface area and strong magnetic moment could be used as magnetically recoverable nanoparticle catalysts. Here we report the development of bimetallic Co-Pd nanoparticles with surface modification by using five capping agents. The nanoparticles were applied to the aerobic oxidation of various alcohols in water as magnetically recoverable and reusable catalysts. 


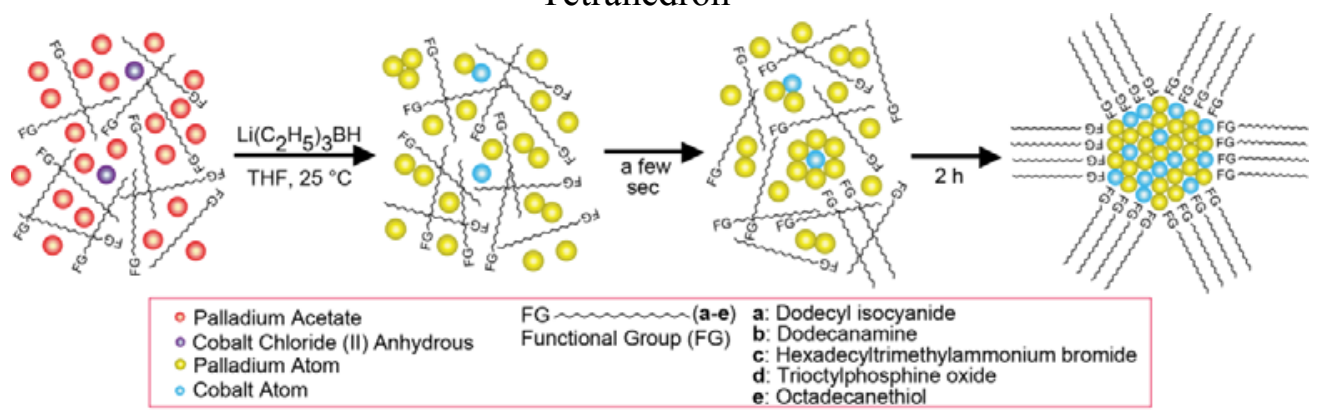

Scheme 1. Preparation of Co-Pd alloy nanoparticles 1a-e with their surface modified by different capping agents a-e.

\section{Results and Discussion}

Bimetallic Co-Pd alloy nanoparticles were prepared by the reduction of $\mathrm{CoCl}_{2}$ and $\mathrm{Pd}(\mathrm{OAc})_{2}$ in the molar ratio of $1: 1$, with lithium triethylborohydride in THF solution in the presence of the capping agents 1a-e (dodecyl isocyanide (a), dodecanamine (b), hexadecyltrimethylammonium bromide (c), trioctylphosphine oxide (d), octadecanethiol (e)) as shown in Scheme 1. The nanoparticles 1a-e were characterized by transmission electron microscope (TEM), and inductively coupled plasma (ICP) and X-ray photoelectron spectroscopy (XPS) to reveal their structures. Figure 1 shows representative TEM images and the distribution of the nanoparticles. All the nanoparticles 1a-e have a small average diameter with narrow distribution (1a: $2.9 \pm 0.8 \mathrm{~nm}, \mathbf{1 b}: 2.9 \pm 0.6 \mathrm{~nm}, \mathbf{1 c}: 4.1 \pm 0.7 \mathrm{~nm}$, 1d: $4.1 \pm 0.7 \mathrm{~nm}, 1 \mathrm{e}: 3.0 \pm 0.4 \mathrm{~nm}$ ) (Table 1). The ratio of Co and Pd was determined by ICP analysis to be 1:1 (Table 1). Table 2 shows XPS analysis data of the nanoparticles 1a-d for Pd and Co surface state where the binding energy of $\mathrm{Pd} 3 \mathrm{~d}_{5 / 2}$ was 1a: 335.1 eV, 1b: $335.3 \mathrm{eV}, \mathbf{1 c}: 335.5 \mathrm{eV}$ and $\mathbf{1 d}: 335.2 \mathrm{eV}$, respectively. These values were close to that of bulk Pd $(334.9 \mathrm{eV}) .{ }^{18}$ In these nanoparticles 1a-d, the binding energy of the nanoparticles modified with dodecyl isocyanide a was the lowest and that of the nanoparticles modified with hexadecyltrimethylammonium bromide $\mathbf{c}$ was the highest. These differences are thought to be caused by surface modification of the capping agents that changed the surface state. On the other hand, the binding energy of Co 2p $p_{3 / 2}$ was 1a: $781.0 \mathrm{eV}, \mathbf{1 b}: 779.9 \mathrm{eV}, \mathbf{1 c}: 780.3 \mathrm{eV}$ and $\mathbf{1 d}$ : $780.7 \mathrm{eV}$, which were close to that of bulk $\mathrm{CoO}(780.0 \mathrm{eV}) .^{18}$ The results suggest that $\mathrm{Co}$ atoms on the surface were airoxidized.
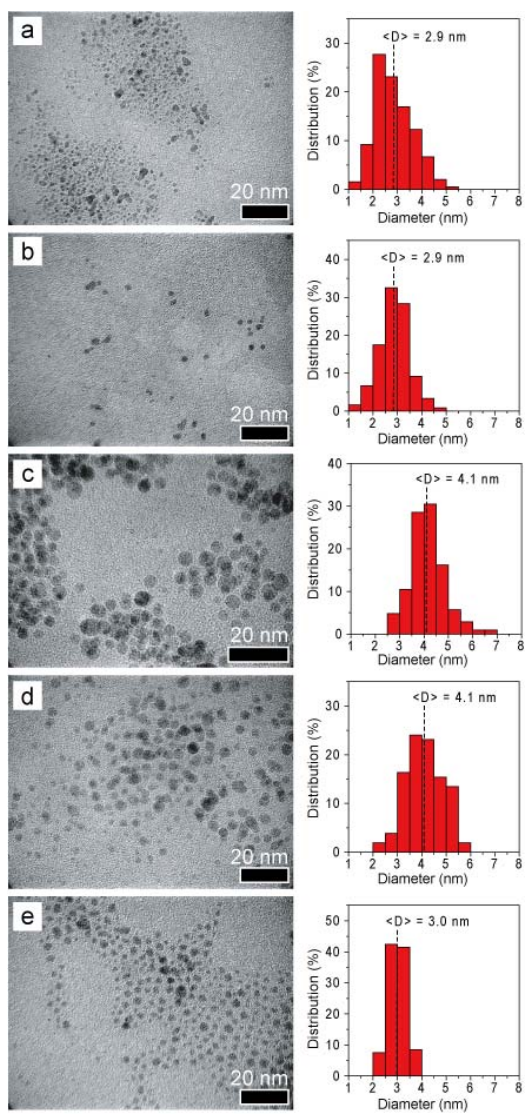

Figure 1 TEM images and the distribution of Co-Pd alloy nanoparticles $\mathbf{1 a}-\mathbf{e}$ with their surface modified by different capping agents a-e. The black scale bars are $20 \mathrm{~nm}$.

Table 1. Structural information of Co-Pd alloy nanoparticles 1a-e

\begin{tabular}{|l|l|l|l|}
\hline $\begin{array}{l}\text { Co-Pd alloy } \\
\text { nanoparticle }\end{array}$ & $\begin{array}{l}\text { Diameter } \pm \text { SD } \\
(\mathrm{nm})\end{array}$ & Co atomic \% & Pd atomic \% \\
\hline 1a & $2.9 \pm 0.8$ & 44 & 56 \\
\hline 1b & $2.9 \pm 0.6$ & 50 & 50 \\
\hline 1c & $4.1 \pm 0.7$ & 49 & 51 \\
\hline 1d & $4.1 \pm 0.7$ & 49 & 51 \\
\hline 1e & $3.0 \pm 0.4$ & 50 & 50 \\
\hline
\end{tabular}


Table 2 Binding energies of Co-Pd alloy nanoparticles 1a-d in the $\mathrm{Pd} 3 \mathrm{~d}_{5 / 2}$ and Co $2 \mathrm{p}_{3 / 2}$ obtained from XPS spectra regions $^{[\mathrm{a}]}$

\begin{tabular}{|l|l|l|}
\hline Co-Pd alloy nanoparticle & $\mathrm{Pd}_{3} \mathrm{~d}_{5 / 2}(\mathrm{eV})$ & Co $2 \mathrm{p}_{3 / 2}(\mathrm{eV})$ \\
\hline 1a & 335.1 & 781.0 \\
\hline 1b & 335.3 & 779.9 \\
\hline 1c & 335.5 & 780.3 \\
\hline 1d & 335.2 & 780.7 \\
\hline
\end{tabular}

${ }^{[\mathrm{a}]}$ The spectra are normalized with $\mathrm{C} 1 \mathrm{~s} 284.6 \mathrm{eV} .{ }^{18}$
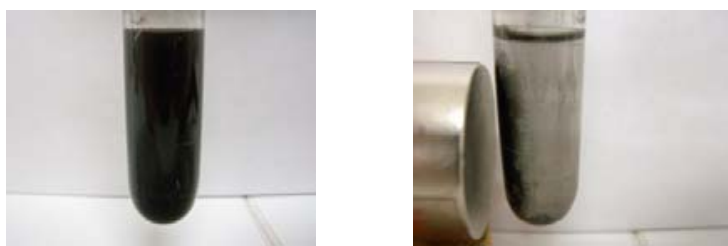

Figure 2. The aerobic oxidation of 2-octanol (2a) in water with Co-Pd alloy nanoparticles 1a. Left: the mixture after the reaction. Right: separation of the Co-Pd alloy nanoparticles from the reaction mixture with a $\mathrm{Nd}$ magnet. The recovery of magnetic catalysts took $10-15 \mathrm{~min}$.

For the Co-Pd alloy nanoparticles 1a-e having different capping agents, the aerobic oxidation of 2-octanol (2a) was investigated in water (Table 3). Thus, the oxidization of $\mathbf{2 a}$ was carried out with the nanoparticles $1 \mathbf{a}-\mathbf{d}(20 \mathrm{~mol} \% \mathrm{Pd})$ in water at $60{ }^{\circ} \mathrm{C}$ for $24 \mathrm{~h}$ under oxygen atmospheric conditions ( $\left.1 \mathrm{~atm}\right)$. The nanoparticles 1a (modified with dodecyl isocyanide a) showed the highest catalytic activity, giving 2-octanone (3a) with $88 \%$ yield (entry 1). The reaction with $\mathbf{1 b}-\mathbf{d}$ under similar conditions gave 3a with $78-64 \%$ yield (entries $6-8$ ). The catalytic activity was different regardless of similar diameter [1a vs. $1 \mathbf{b}(\mathbf{1 a}: 2.9 \pm$ $0.8 \mathrm{~nm}$ (3a: $88 \%$ yield), 1b: $2.9 \pm 0.6 \mathrm{~nm}$ (3a: $78 \%$ yield)), and 1c vs. 1d (1c: $4.1 \pm 0.7 \mathrm{~nm}$ (3a: $71 \%$ yield), 1d: $4.1 \pm 0.7 \mathrm{~nm}$ (3a: $64 \%$ yield))]. The difference is thought to be caused by differences of capping agents: considering the surface state of the nanoparticles 1a, the back donation of Pd to the isocyanide group could serve to enhance catalytic activity. ${ }^{19}$ The nanoparticles 1d, modified with trioctylphosphine oxide, should have strong affinity to $\mathrm{Pd}$ nanoparticles, thus degrading the catalytic activity. ${ }^{20}$ The nanoparticles 1e modified with octadekanethiol showed no catalytic activity because the thiol group capped their surface too strongly (entry 9).

Table 3. The aerobic oxidation of 2-octanol (2a) in water with Co-Pd alloy nanoparticles $1 \mathbf{a}-\mathbf{e}^{[\mathrm{a}]}$

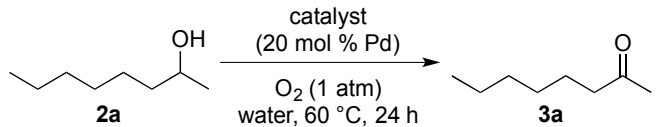

\begin{tabular}{|l|l|l|}
\hline Entry & Catalyst & Yield (\%) \\
\hline 1 & $\mathbf{1 a}$ & 88 \\
\hline 2 & $\mathbf{1 a}^{[\mathrm{b}]}$ & 91 \\
\hline 3 & $\mathbf{1 a}^{[\mathrm{c}]}$ & 80 \\
\hline 4 & $\mathbf{1 a}^{[\mathrm{d}]}$ & 18 \\
\hline 5 & $\mathbf{1 a}^{[\mathrm{e}]}$ & 38 \\
\hline
\end{tabular}

\begin{tabular}{|l|l|l|}
\hline 6 & 1b & 78 \\
\hline 7 & 1c & 71 \\
\hline 8 & 1d & 64 \\
\hline 9 & 1e & $<1$ \\
\hline \multicolumn{2}{|l|}{${ }^{[a] ~ c o n d i t i o n s: ~ c a t a l y s t ~(20 ~ m o l \% ~ P d), ~ 2 a ~(0.2 ~ m m o l), ~ w a t e r ~(2 ~}$}
\end{tabular}
$\mathrm{mL}), \mathrm{O}_{2}(1 \mathrm{~atm}), 60^{\circ} \mathrm{C}, 24 \mathrm{~h} ;{ }^{[\mathrm{b}} 2 \mathrm{nd}$ use of $1 \mathrm{a} ;{ }^{[\mathrm{c}]} 3 \mathrm{rd}$ use of $\mathbf{1 a}$; ${ }^{[\mathrm{d}]} 4$ th use of $1 \mathbf{a} ;{ }^{[\mathrm{e}]} 5$ th use of $1 \mathbf{a}$.

Table 4. The aerobic oxidation of alcohols $\mathbf{2 b}-\mathbf{j}$ or $\mathbf{4}$ in water with the Co-Pd alloy nanoparticles $\mathbf{1} \mathbf{a}^{[\mathrm{a}]}$

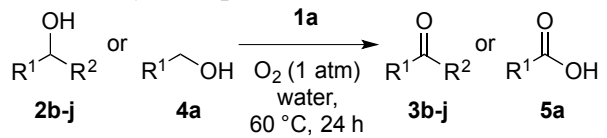

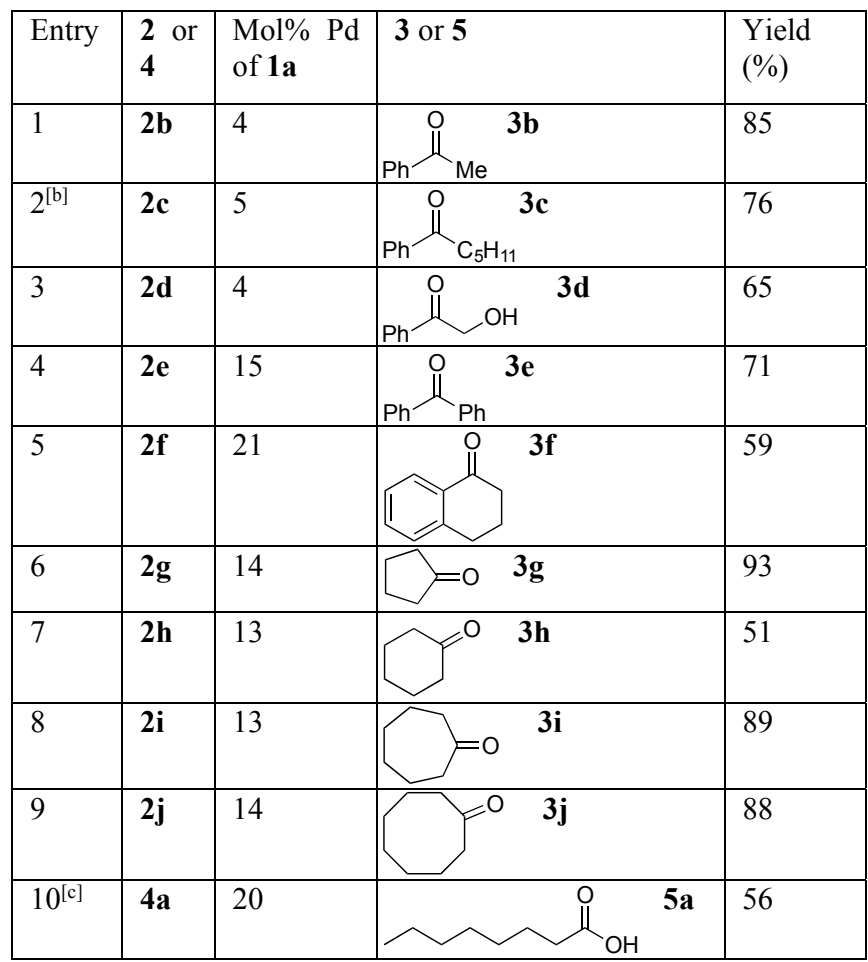

${ }^{[a]}$ All reactions were carried out with 1a, alcohols $(0.2 \mathrm{mmol})$ in water $(2 \mathrm{~mL})$ at $60{ }^{\circ} \mathrm{C}$ for $24 \mathrm{~h}$ under atmospheric oxygen; ${ }^{[b]}$ 20 mmol scale; ${ }^{[c]} \mathrm{K}_{2} \mathrm{CO}_{3}$ (1 mol equiv) was added.

The nanoparticle catalyst 1a showing the highest catalytic activity was applied to the aerobic oxidation of various secondary alcohols $\mathbf{2 b}-\mathbf{j}$ and a primary alcohol $\mathbf{4 a}$ in water (Table 4). The oxidation of reactive benzylic alcohols $\mathbf{2 b}-\mathbf{d}$ gave $\mathbf{3 b} \mathbf{b}-\mathbf{d}$ with $65-$ $75 \%$ yield by use of $4-5 \mathrm{~mol} \% \mathrm{Pd}$ of the catalyst $1 \mathrm{a}$ (entries $1-$ 3 ). The selective oxidation of an alcohol $\mathbf{2 d}$ which consists of benzylic and aliphatic alcohol moieties was achieved to give the benzylic ketone 3d with 65\% yield (entry 3). Sterically hindered benzylic alcohols $2 \mathrm{e}$ and $\mathbf{2 f}$ required a larger amount of the catalyst (entries 4 and 5). The nanoparticle catalyst 1a promoted the aerobic oxidation of less reactive alicyclic alcohols, cyclopentanol (2g), cycloheptanol (2i) and cyclooctanol (2j) to give $\mathbf{3 g}, \mathbf{3 i}$, and $\mathbf{3 j}$ with $93 \%, 89 \%$ and $88 \%$ yield, respectively (entries 6, 8, and 9), while cyclohexanol (2h) was converted to $3 \mathrm{~h}$ with $51 \%$ yield (entry 7 ). The reaction of a primary aliphatic alcohol 1-octanol (4a) gave octanoic acid (5a) with 56\% yield 
(entry 10) in which the resulting carboxylate could act as a capping agent of nanoparticles to decrease the catalytic activity. ${ }^{20}$

The nanoparticle catalyst 1a for the oxidation of 2-octanol (2a) was employed for magnetic recovery and reuse experiments (Table 1, entries 1-5). After completion of the first reaction, the catalyst 1a was magnetically recovered with a $\mathrm{Nd}$ magnet (Figure 2 ), and then the $2 \mathrm{nd}, 3 \mathrm{rd}$, 4th, and 5th reactions were carried out with the recovered catalyst under similar conditions to give $91 \%$, $80 \%, 18 \%$ and $38 \%$ yield of 2 -octanone (3a), respectively. Figure 3 shows a representative TEM image after the 4 th reaction. The diameter of the nanoparticle catalyst 1a increased from $2.9 \pm 0.8$ $\mathrm{nm}$ (before use) to $5.0 \pm 1.1 \mathrm{~nm}$ (after the 4 th reaction). Leaching of $\mathrm{Co}$ and Pd species after the 1 st reaction was checked with ICP analysis. Leaching of Co and Pd was only $9 \times 10^{-3} \mathrm{~mol} \%$ and $8 \times$ $10^{-6} \mathrm{~mol} \%$, respectively, whose ratio of Co to $\mathrm{Pd}$ is $>99.9:<0.1$. This result can be explained with an oxidizing ability of $E_{\text {ox }}$ : $0.951 \mathrm{~V}$ of $\mathrm{Pd}(0) / \mathrm{Pd}(\mathrm{II})$ and $E_{\text {ox }}:-0.28 \mathrm{~V}$ of $\mathrm{Co}(0) / \mathrm{Co}(\mathrm{II})$ with reference to the standard hydrogen electrode. $\operatorname{Co}(0)$ should be readily oxidized by $\mathrm{Pd}(\mathrm{II})$ to give ionic $\mathrm{Co}(\mathrm{II})$ that could be leached out. The resulting $\operatorname{Pd}(0)$ could aggregate with initial nanoparticles to provide larger nanoparticles with wider distribution (2-8 nm).

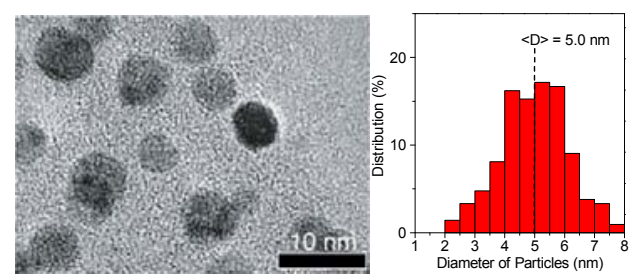

Figure 3. Representative TEM image of Co-Pd alloy nanoparticles 1a after the 4 th run in the aerobic oxidation in water. The black scale bar is 10 nm.

\section{Conclusion}

In conclusion, we prepared and characterized bimetallic Co$\mathrm{Pd}$ alloy nanoparticles modified with various capping agents whose atomic concentrations were finely controlled. We bifunctionalized Co-Pd alloy nanoparticles with catalytic activity by $\mathrm{Pd}$ as well as magnetic recoverability by Co simultaneously. The Co-Pd alloy nanoparticle catalyst showed catalytic activity for the aerobic oxidation of various alcohols in water with recyclability under mild conditions.

\section{Experimental Section}

\subsection{General Methods}

The aerobic oxidation of alcohols was performed in a glass tube under oxygen gas at atmospheric pressure in a balloon. THF was distilled from $\mathrm{Na}$ /benzophenone. Water was deionized with a Millipore system. ${ }^{1} \mathrm{H}$ NMR $(500 \mathrm{MHz})$ and ${ }^{13} \mathrm{C}\left\{{ }^{1} \mathrm{H}\right\}$ NMR (126 $\mathrm{MHz}$ ) spectra were measured with a JNM ECA-500 spectrometer. ${ }^{1} \mathrm{H}$ NMR chemical shifts are reported relative to tetramethylsilane (TMS, $0.00 \mathrm{ppm}$ ) as an internal standard. The ${ }^{13} \mathrm{C}\left\{{ }^{1} \mathrm{H}\right\}$ NMR chemical shifts are reported relative to $\mathrm{CDCl}_{3}$ (77.0 ppm). The GC-MS was measured by an Agilent 7890A $\mathrm{GC} / \mathrm{MS}$ detector that was used with $o$-xylene or mesitylene as an internal standard for determining the GC yield. TEM images were obtained by using a transmission electron microscope (JEOL JEM-2100F) operated at $200 \mathrm{kV}$. TEM samples were prepared by placing a hexane suspension of nanoparticles on carbon-coated $\mathrm{Cu}$ grids, and allowed to dry at room temperature. XPS spectra were measured by ESCALAB 250 (Thermo Scientific). Elemental analyses of Co and Pd were carried out by inductively coupled plasma spectroscopy using Shimadzu ICPS8100 equipment by the chemical analysis team in RIKEN. Magnet recovery of catalysts was performed with a $\mathrm{Nd}$ magnet (ca 0.34 T). Capping agents, dodecanamine (b), hexadecyltrimethylammonium bromide (c) and trioctyl phosphine oxide (d) were purchased from Aldrich and Wako. Dodecyl isocyanide (a) was prepared according to the literature ${ }^{21}$. The alcohols, 2-octanol (2a), 1-phenylethanol (2b), diphenylmethanol (2c), 1-phenyl-1-hexanol (2d), 1,2,3,4tetrahydro-1-naphthol (2e), 1-phenyl-1,2-ethanediol (2f), cyclopentanol (2g), cyclohexanol (2h), cycloheptanol (2i), cyclooctanol (2j), 1-octanol (4a), palladium acetate, anhydrous cobalt chloride (II) and lithium triethylborohydride were purchased from Aldrich, TCI, Wako and ACRŌS Organics.

All oxidation products are known compounds whose CA registry numbers are provided as below:

3a: 111-13-7, 3b: 98-86-2, 3c: 942-92-7, 3d: 582-24-1, 3e: 119-61-9, 3f: 529-34-0, 3g: 120-92-3, 3h: 108-94-1, 3i: 502-421, 3j: 502-49-8, 5a: 124-07-2.

\subsection{General Procedure for Co-Pd Alloy Nanoparticles}

The nanoparticles of Co-Pd alloy modified with capping agents a-e were prepared similarly to Co-Pd alloy nanoparticles. ${ }^{14} \mathrm{~A}$ mixture of $\mathrm{Pd}(\mathrm{OAc})_{2}(0.25 \mathrm{mmol})$, anhydrous $\mathrm{CoCl}_{2}(0.25 \mathrm{mmol})$, and capping agents a-e $(0.5 \mathrm{mmol}(\mathbf{a}-\mathbf{d})$ or $0.35 \mathrm{mmol}(\mathbf{e}))$ was dissolved in $10 \mathrm{~mL}$ of freshly distilled dry THF under nitrogen. To this solution lithium triethylborohydride solution in THF (1 M, $5 \mathrm{~mL})$ was rapidly added in one portion at $24{ }^{\circ} \mathrm{C}$. The resulting suspension was stirred at $24{ }^{\circ} \mathrm{C}$ for $2 \mathrm{~h}$ under nitrogen. The resulting nanoparticles were collected by centrifugation and purified with ethanol to give the corresponding nanoparticles $\mathbf{1 a}-\mathbf{e}$ as a suspension in ethanol (the concentrations of Co and Pd in ethanol were determined by ICP analysis) (CAUTION! Dried nanoparticles are ignitable.).

\subsection{General Procedure for Catalytic Aerobic Oxidation of Primary Alcohols in Water}

A mixture of magnetic nanoparticles 1a-e (suspension in ethanol; $\sim 0.16 \mathrm{M} \mathrm{Co-Pd})$, primary alcohol $(0.2 \mathrm{mmol})$, and potassium carbonate $(0.2 \mathrm{mmol})$ in water $(2 \mathrm{~mL})$ was stirred at 60 ${ }^{\circ} \mathrm{C}$ under oxygen gas at atmospheric pressure. After being cooled, the mixture was washed with ether, and acidified with $5 \%$ hydrochloric acid. The mixture was extracted with ethyl acetate $(5 \times 1 \mathrm{~mL})$. The extract was dried over magnesium sulfate. The resulting solution was analyzed by GC and GC-MS (with Mass Spectral Reference Database). The yield of the product was determined by $\mathrm{GC}$ with an internal standard (o-xylene).

\subsection{General Procedure for Catalytic Aerobic Oxidation of Secondary Alcohols in Water}

A mixture of magnetic nanoparticles 1a (suspension in ethanol) and secondary alcohol $(0.2 \mathrm{mmol})$ in water $(2 \mathrm{~mL})$ was stirred at $60{ }^{\circ} \mathrm{C}$ under oxygen gas at atmospheric pressure. After it was cooled, the mixture was extracted with ethyl acetate $(5 \times 1$ $\mathrm{mL}$ ). The extract was analyzed by GC and GC-MS (with Mass Spectral Reference Database). The yield of the product was 
determined by GC with an internal standard (o-xylene or mesitylene).

\section{Acknowledgments}

We thank RIKEN for XPS (Dr. Aiko Nakao), TEM (Ms. Tomoka Kikitsu) and ICP-AES (Ms. Chieko Kariya) analyses. We gratefully acknowledge financial support from JST ACT-C, JST CREST, JSPS (\#24550126, \#20655035 and \#2105), Takeda Science Foundation, the Naito Foundation, and RIKEN. Y.I. was supported by JPSP Fellowship.

\section{References and notes}

1. For recent reviews, see: (a) Astruc, D.; Lu, F.; Aranzaes, R. Angew. Chem. Int. Ed. 2005, 44, 7852; (b) Mallat, T.; Baiker, A. Chem. Rev. 2004, 104, 3037; (c) Anderson, R. A.; Griffin, K.; Johnson, P.; Alsteres, P. L. Adv. Synth. Catal. 2003, 345, 517-523.

2. (e) Uozumi, Y.; Yamada, Y. M. A. Chem. Rec. 2009, 9, 51; (b) Yamada, Y. M. A.; Arakawa, T.; Hoche, H.; Uozumi, Y. Chem. Asian J. 2009, 4, 1092; (c) Yamada, Y. M. A.; Arakawa, T.; Hoche, H.; Uozumi, Y. Angew. Chem. Int. Ed. 2007, 46, 704; (d) Nakao, R.; Rhee, Y.; Uozumi, Y. J. Organomet. Chem. 2007, 692,

3. Mori, K.; Hara, T.; Mizugaki, T.; Ebitani, K.; Kaneda, K. J. Am. Chem. Soc. 2004, 126, 10657.

4. (a) ten Brink, G.; Arends, I. W. C. E.; Sheldon, R. A. Science 2000, 287, 1636; (b) ten Brink, G.; Arends, I. W. C. E.; Hoogenraad, M.; Verspui, G.; Sheldon, R. A. Adv. Synth. Catal. 2003, 345, 1341; (c) ten Brink, G.; Arends, I. W. C. E.; Hoogenraad, M.; Verspui, G.; Sheldon, R. A. Adv. Synth. Catal. 2003, 345, 497.

5. Abad, A.; Concepción, P.; Corma, A.; García, H. Angew. Chem. Int. Ed. 2005, 44, 4066.

6. Tsunoyama, H.; Tsukuda, T.; Sakurai, H. Chem. Lett. 2007, 36, 212.

7. Polshettiwar, V.; Varma, R. S. Org. Biomol. Chem. 2009, 7, 37.

8. Lang, L.; Li, B.; Liu, W.; Li, X.; Xu, Z. Chem. Lett. 2009, 38, 806.
9. Jacinto, M. J.; Santos, O. H. C. F.; Jardim, R. F.; Landers, R.; Rossi, L. M. Appl. Catal. A: General 2009, 360, 177.

10. Oliveira, R. L.; Kiyohara, P. K.; Rossi, L. M. Green. Chem. 2010, $12,144$.

11. Kotani, M.; Koide, T.; Yamaguchi, K.; Mizuno, N. Green. Chem. 2006, 8, 735 .

12. Baruwati, B.; Polshettiwar, V.; Varma, R. S. Tetrahedron Lett. 2009, 50, 1215

13. (a) Mallat, T.; Baiker, T. Chem. Rev. 2004, 104, 3037; (b) Astruc, A.; Aranzaes, J. R. Angew. Chem. Int. Ed. 2005, 44, 7852; (c) Sifelt, J. H. Acc. Chem. Res. 1977, 10, 15.

14. Tu, W.; Fukui, K.; Miyazaki, A.; Enoki, T. J. Phys. Chem. B 2006, 110, 20895.

15. (a) Ito, Y.; Miyazaki, A.; Fukui, K.; Valiyaveettil, S.; Yokoyama, T.; Enoki, T. J. Phys. Soc. Jpn. 2008, 77, 103701; (b) Ito, Y.; Takai, K.; Enoki, T. J. Phys. Chem. C. 2011, 115, 8971; (c) Ito, Y.; Miyazaki, A.; Takai, K.; Sivamurugan, V.; Maeno, T.; Kadono, T.; Kitano, M.; Ogawa, Y.; Nakamura, N.; Hara, M.; Valiyaveettil, S.; Enoki. T. J. Am. Chem. Soc., 2011, 133, 11470; (d) Ito, Y.; Takai, K.; Miyazaki, A.; Sivamurugan, V.; Kiguchi, M.; Ogawa, Y.; Nakamura, N.; Valiyaveettil, S.; Tada, T.; Watanabe, S.; Enoki, T. Phys. Chem. Chem. Phys., 2014, 16, 288.

16. Ito, Y.; Miyazaki, A.; Valiyaveettil, S.; Enoki, T. J. Phys. Chem. C 2010, 114, 11699.

17. Nunomura, N.; Hori, H.; Teranishi, T.; Miyake, M.; Yamada, S. Phys. Lett. A 1998, 249, 524.

18. Wagner, C. D.; Ringgs, W. M.; Davis, L. E.; Moulder, J. F.; Muilenberg, G. E. Handbook of X-Ray Photoelectron Spectroscopy, Eden Prairie, MN, USA, 1979.

19. Murphy, K. L.; Tysoe, W. T.; Bennett, D. W. Langmuir 2004, 20, 1732.

20 (a) Jun, Y.; Choi, J.; Cheon, J. Angew. Chem. Int. Ed. 2006, 45, 3414; (b) Corthey, G.; Rubert, A. A.; Picone, A. L.; Casillas, G.; Giovanetti, L. J.; Ramallo-López, J. M.; Zelaya, E.; Benitez, G. A.; Requejo, F. G.; José-Yacamán, M.; Salvarezza, R. C.; Fonticelli, M. H. J. Phys. Chem. C 2012, 116, 9830.

21. Miller, D. G.; Trenbeath, S.; Sih, C. J. Tetrahedron Lett. 1976, 17, 1637. 


\section{Graphical Abstract}

To create your abstract, type over the instructions in the template box below.

Fonts or abstract dimensions should not be changed or altered.

\section{Bimetallic Co-Pd Alloy Nanoparticles as}

Magnetically Recoverable Catalysts for the

Leave this area blank for abstract info.

Aerobic Oxidation of Alcohols in Water

Yoshikazu Ito, ${ }^{a, b}$ Hidetoshi Ohta, ${ }^{a}$ Yoichi M. A. Yamada, ${ }^{a *}$ Toshiaki Enoki, ${ }^{b}$ and Yasuhiro Uozumi ${ }^{a, c^{*}}$

${ }^{a}$ RIKEN Center for Sustainable Resource Science, Wako, Saitama 351-0198, Japan

${ }^{b}$ Department of Chemistry, The Graduate School of Science and Engineering, Tokyo Institute of Technology, Ookayama, Tokyo 152-8551, Japan

${ }^{c}$ Institute for Molecular Science, Okazaki, Aichi 444-8787, Japan

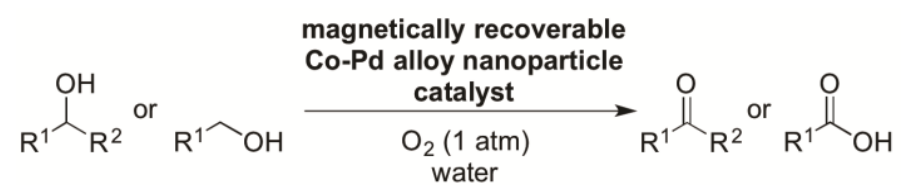

